\title{
Effect of (meth)acrylates on in situ visible light polymerization of dimethacrylamide
}

\author{
YULING XU $\mathrm{XU}^{1,2}$ and DONG $\mathrm{XIE}^{2, *}$ (D) \\ ${ }^{1}$ College of Chemical and Environmental Engineering, Wuhan Polytech University, Wuhan, Hubei 430062, People's \\ Republic of China \\ ${ }^{2}$ Department of Biomedical Engineering, Indiana University_Purdue University at Indianapolis, Indianapolis 46202, USA \\ *Author for correspondence (dxie@iupui.edu)
}

MS received 10 March 2017; accepted 12 December 2017; published online 25 July 2018

\begin{abstract}
This study reports in situ visible light copolymerization of a synthesized dimethacrylamide with the selected (meth)acrylates. The effects of these selected comonomers with different functional groups on the polymerization rate, degree of conversion, gel time and compressive strength were investigated. The results showed that in situ copolymerization of the dimethacrylamide with the comonomers having an electron-withdrawing and/or acrylate group could significantly increase the polymerization rate, degree of conversion and compressive strength. Contrarily, an electron-donating group on either carbon-carbon double bond or ester linkage could slow down the polymerization. In comparison, its methacrylate counterpart, triethylene glycol dimethacrylate-based system did not show a clear pattern. The formed H-bonds between (meth)acrylamide and organic acid groups may be responsible for higher compressive strengths. Within the limitation of this study, it is concluded that in situ polymerization of dimethacrylamide under visible light can be accelerated by copolymerization with monomers having electron-withdrawing and/or acrylate groups and vice versa.
\end{abstract}

Keywords. Dimethacrylamide; visible light polymerization; degree of conversion; compressive strength.

\section{Introduction}

Visible light or redox-initiated polymerizations are very popular and useful in many biomedical applications [1,2]. Most of the monomers or polymers used for these applications are methacrylates or acrylates or their derivatives [3], because ester linkage-based vinyl groups demonstrate a fast in situ polymerization rate and are user friendly [4]. However, recent studies found that methacrylates/acrylates, dimethacrylates/diacrylates or their derivatives are vulnerable to oral esterases [5,6] and/or highly acidic environment [7] due to instability of ester linkages [5-7]. The potential degradation of dimethacrylates/diacrylates-crosslinked polymer networks caused by esterases and/or acidic environment is particularly detrimental to restoration because the degraded polymer networks could cause restoration failure. As an alternative, acrylamide and methacrylamide-based monomers were hydrolytically stable in acidic environment and in the presence of esterase [8]. However, these alternative monomers or oligomers are still not popular because of their slow in situ polymerization kinetics [9]. It was reported that in situ polymerization of diacrylamide, $\mathrm{N}, \mathrm{N}^{\prime}$-diethyl-1,3-bis(acrylamido)propane (DEBAAP) alone showed lower polymerization rate as well as lower degree of conversion under visible light illumination as compared to methacrylate- or acrylate-based systems $[9,10]$. It was also found that acrylamide containing a phosphoric acid could increase the polymerization rate and degree of conversion of DEBAAP when they were copolymerized with each other [10]. Furthermore, it was found that the polymerization rate and degree of conversion were increased with DEBAAP in the presence of non-polymerizable organic phosphoric acid [11]. Then, it was concluded that the light polymerization of acrylamide monomer, such as DEBAAP could be enhanced by the increase of medium polarity brought by the presence of phosphoric acid groups [11]. Our preliminary study on dimethacrylamide also indicated that the monomer itself was hard to be homo-polymerized under blue light or even visible light in the presence of camphorquinone and $\mathrm{N}, \mathrm{N}^{\prime}$-dimethylaminoethyl methacrylate light-initiation system [12]. However, it was found that the polymerization was much faster, if we incorporate acrylic acid into the system. To extend the preliminary study, we proposed to investigate the effect of the selected (meth)acrylate monomers, which are often used in biomedical/dental applications on in situ light polymerization of dimethacrylamide and tried to find some correlation between dimethacrylamide and the selected (meth)acrylate monomers, so as to make diacrylamide or dimethacrylamide to be more applicable in biomedical research. Therefore, the purpose of this study was to investigate the effect of the selected (meth)acrylates on in situ polymerization rate, degree of conversion and compressive strength of the newly synthesized dimethacrylamide with visible light initiation. 


\section{Experimental}

\subsection{Materials}

1,8-Diamino-3,6-dioxaoctane (DADO), methacrylic anhydride (MAAn), triethylamine, acrylic acid (AA), methacrylic acid (MAA), ethylene glycol methacrylate phosphate (EGMAP), hydroxyethyl acrylate (HEA), hydroxyethyl methacrylate (HEMA), methyl acrylate (MA), methyl methacrylate (MMA), triethylene glycol dimethacrylate (TEGDMA), camphorquinone, N,N-dimethylaminoethyl methacrylate, acetone and diethyl ether were used as received from Sigma-Aldrich Chemical Co. (Milwaukee, WI, USA) without further purification.

\subsection{Synthesis and characterization}

2.2a Synthesis: Synthesis of dioxaoctane dimethacrylamide (DDM) is described below. In summary, to a flask containing DADO $(0.05 \mathrm{~mol})$ and acetone in an ice-bath, MAAn $(0.11 \mathrm{~mol})$ was added dropwise while stirring. Then, the reaction was run at room temperature overnight. The solution was concentrated with a rotary evaporator, followed by dissolving in ether, washing with sodium bicarbonate solution and brine, drying with anhydrous $\mathrm{MgSO}_{4}$ and then, concentrating in vacuo to obtain the purified product DDM (yield $>87 \%$ ). The structure of DDM is shown in figure 1 .

2.2b Characterization: The synthesized oligomer was characterized by Fourier transform infrared (FTIR) and nuclear magnetic resonance (NMR) spectroscopies. The

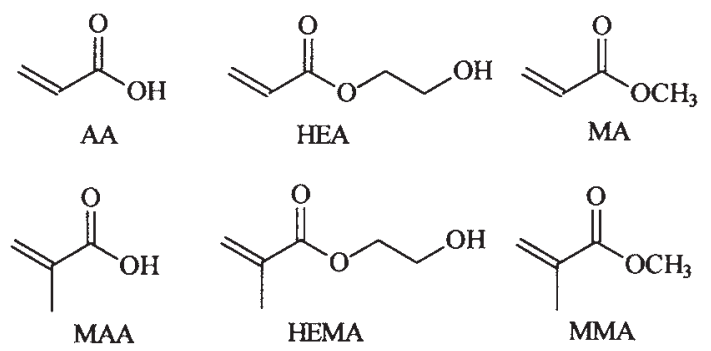<smiles>C=C(C)C(=C)C(=O)OCCOCCOCCNC(=O)C(=C)C(=O)OCCOCCOC(=O)C(=C)C</smiles>

Figure 1. Schematic chemical structures of the monomers used in the study. proton NMR ( $\left.{ }^{1} \mathrm{HNMR}\right)$ spectra were obtained on a $500 \mathrm{MHz}$ Bruker NMR spectrometer (Bruker Avance II, Bruker BioSpin Corporation, Billerica, MA) using deuterated dimethyl sulfoxide as solvent and FTIR spectra were obtained on a FTIR spectrometer (Mattson Research Series FT/IR 1000, Madison, WI).

\subsection{Evaluation}

2.3a Formulation: The resin liquid was formulated with DDM or TEGDMA, commercial monomers, $1.5 \%$ camphorquinone (photo-initiator, wt/wt) and $3.0 \% \mathrm{~N}, \mathrm{~N}-$ dimethylaminoethyl methacrylate (activator), where DDM or TEGDMA was either formulated alone or mixed with a comonomer in a ratio of 50:50 (wt/wt) [12].

2.3b Polymerization conversion and gel time: Polymerization conversion was evaluated by measuring degree of monomer conversion via a FTIR spectrometer, following the published protocol [13]. Briefly, liquid resin sample was cast within two $\mathrm{KBr}$ crystals, followed by illuminating with a visible light device (Tungsten halogen light source, $250 \mathrm{~W}$, Tricure 2000, Dentsply, York, PA). Then, the KBr crystal sandwich was mounted on the FTIR sample holder and scanned to acquire a FTIR spectrum. The peak areas on the absorbance spectra of the samples were used to determine degree of conversion (DC). The areas under the peaks at 1617 and $3331 \mathrm{~cm}^{-1}$, assigned to vinyl $\mathrm{C}=\mathrm{C}$ and amide $\mathrm{H}$ stretching for the DDM-based resin, and at 1637 and $1722 \mathrm{~cm}^{-1}$, to vinyl $\mathrm{C}=\mathrm{C}$ and ester for the TEGDMA-based resin, were used to calculate DC. DC was determined using the equation of $1-\left[\left(A_{\mathrm{C}=\mathrm{C} \text {, cured }} / A_{\text {amide or ester,cured }}\right) /\left(A_{\mathrm{C}=\mathrm{C} \text {, uncured }} /\right.\right.$ $\left.\left.A_{\text {amide or ester uncured }}\right)\right] \times 100$, where $A$ is the measured peak area. The gel time was estimated by visual observation, where the resin or composite sample starts to shrink under the light illumination.

2.3c Strength evaluation: Cylindrical specimens were fabricated at room temperature according to the published protocol [12]. Briefly, the thoroughly mixed resin liquid was placed into a cylindrical glass mould with dimensions of $4 \mathrm{~mm}$ in diameter and $8 \mathrm{~mm}$ in length. Then, the specimens were exposed to visible light for $10 \mathrm{~min}$ to ensure a complete polymerization. The sample sizes were $n=6-8$ for each formulation. The compressive strength (CS) test was performed on a screw-driven mechanical tester (QTest QT/10, MTS Systems Corporation, Eden Prairie, MN, USA) with a crosshead speed of $1 \mathrm{~mm} \min ^{-1}$ [14]. CS was calculated using an equation, $\mathrm{CS}=P / \pi r^{2}$, where $P$ is the load at fracture and $r$ the radius of the cylinder. Compressive yield strength (YS), modulus (M), toughness (T) and energy to yield (ETY) were obtained from the stress-strain curves of the CS tests. One-way analysis of variance (ANOVA) with the posthoc Tukey-Kramer multiple range test was used to determine significant differences of the CS among the materials in each 
formulation. A level of $\alpha=0.05$ was used for statistical significance.

\section{Results and discussion}

\subsection{Characterization}

Figure 2a shows the FTIR spectra for DADO, MAAn and DDM. The characteristic peaks are listed below: (1) DADO: 3368 and 1573 ( $-\mathrm{N}-\mathrm{H}$ stretching and deformation) for primary amino group; 1480, 1383 and 1105 (-C-O-C- and $-\mathrm{OCH}_{2}-$ stretching and deformation) for ether group; and 2874, 1354, 819, 698, 624 and 583 (C-H stretching and deformation) for methylene and methyl groups. (2) MAAn: 1783 and 1723 ( $-\mathrm{C}=\mathrm{O}$ stretching) for anhydride group; 1636, 1454 and 1297 ( $-\mathrm{C}=\mathrm{C}-$ stretching and vibration) for methacrylate group; 1050 (-C-O-C-deformation) for anhydride group; and 2930, 1403, 1379, 1181, 1119, 1003, 948, 809 and 641 (C-H stretching and deformation) for methylene and methyl groups. (3) DDM: 3331 and 3086 (-N-H stretching) for amide group; 1719, 1657 and 1534 (-CONH- stretching and deformation) for amide group; 1617, 1454 and 1311 ( $-\mathrm{C}=\mathrm{C}-$ stretching and vibration) for methacrylamide group; 1454 and 1133 (-C-O-C- and $-\mathrm{OCH}_{2}-$ stretching and deformation) for ether group; and 2924, 1412, 1375, 1218, 1007, 931, 808 and 650 (C-H stretching and deformation) for methylene and methyl groups. Disappearance of the broad peak at 3468 for amino group on DADO as well as the strong peaks at 1783 and 1723 for anhydride on MAAn, appearance of strong new peaks at 3331, 1657 and 1534 on DDM, and slightly downshift of the peak at 1636 to 1617 for $\mathrm{C}=\mathrm{C}$ group on $\mathrm{DDM}$ confirmed the formation of the oligomer DDM.

Figure $2 \mathrm{~b}$ shows the ${ }^{1} \mathrm{HNMR}$ spectra for DADO, MAAn and DDM. The chemical shifts (ppm) were: (1) DADO, 3.5, 3.35 and $2.65\left(12 \mathrm{H}\right.$ on $\left.-\mathrm{CH}_{2} \mathrm{CH}_{2}-\right)$ and $1.40\left(4 \mathrm{H}\right.$ on $\left.-\mathrm{NH}_{4}\right)$; (2) MAAn, 6.25 and $6.0(-4 \mathrm{H}$ on $\mathrm{C}=\mathrm{C}), 1.95\left(6 \mathrm{H}\right.$ on $\left.-\mathrm{CH}_{3}\right)$; (3) DDM, 7.95 (2H on -CONH-), 5.65 and 5.35 ( $4 \mathrm{H}$ on $\mathrm{C}=\mathrm{C}), 3.5,3.45$ and $3.25\left(12 \mathrm{H}\right.$ on $\left.-\mathrm{CH}_{2} \mathrm{CH}_{2}-\right)$ and $1.85(6 \mathrm{H}$ on $-\mathrm{CH}_{3}$ ). Significant chemical shifts of $\mathrm{C}=\mathrm{C}$ from 6.25 and 6.0 to 5.65 and 5.35 , disappearance of the chemical shift at $1.4\left(-\mathrm{NH}_{2}\right)$, and formation of all the new chemical shifts on DDM confirmed the successful synthesis of DDM.

\subsection{Evaluation}

So far most of in situ polymerization systems were methacrylate- or acrylate-based. Only a few research reports were focussed on in situ curable methacrylamides or acrylamides, probably due to their slower in situ polymerization $[9,10]$. In this study, a liquid dimethacrylamide-DDM was synthesized and used to formulate a polymerizable and crosslinkable liquid resin system with different acrylates or methacrylates to form the DDM-based resins to study their in situ curing rate and degree of conversion. Prior to the study, we had tried two different commercial available light-curing sources-blue light and visible light, to see if the system can be cured in situ. Blue light is often specified as a wavelength from 450 to $495 \mathrm{~nm}$, whereas visible light from 400 to $700 \mathrm{~nm}$. We found that DDM was relatively hard to be polymerized in situ through a blue light source (wavelength $=465 \mathrm{~nm}$ ), but to be cured relatively easier and faster, using a visible light source with a tungsten-halogen lamp (wavelength $=350$ $1100 \mathrm{~nm}$ ). It took 240 and $95 \mathrm{~s}$ for DDM and AA/DDM resins, respectively, to form gels under blue light. In contrast, with visible light, it took 170 and $40 \mathrm{~s}$ for DDM and AA/DDM resins, respectively, to form gels (table 1). It is possible that the special blue light source used for in situ DDM polymerization of this study has a very narrow and short wavelength, whereas the visible light source has a much broader wavelength spectrum ranging from 350 to $1100 \mathrm{~nm}$, which even covers some UV and near-IR regions. The latter indicates that DDM needs a visible light source to better activate or initiate the in situ polymerization. Therefore, we decided to use visible light to study the proposed system. We evaluated the DC, gel time as well as CS of the DDM-based resins and compared them with those for the TEGDMA-based resins. TEGDMA is a popular and commonly used commercial dimethacrylate, which is used in many biomedical applications. By comparing DDM with TEGDMA in chemical structure (figure 1), obviously two ester linkages are replaced by two amide linkages. To study whether comonomers can affect the in situ light polymerization of DDM, we incorporated acrylates or methacrylates with different pendent functionalities to form the DDM- and TEGDMA-based systems. Polymerization rate was used to measure how fast the reaction would start under visible light illumination, whereas DC was used to investigate how long it would take to complete the reaction. Gel time was used to evaluate when the light initiation would start, while the material was in a practical form for CS evaluationcylinder. CS was used to evaluate how strong the formed resins would be after light illumination, which is another indication of degree of conversion. The details are discussed below.

Figure 3 shows the conversion curves of the DDM- and TEGDMA-based resins vs. time. From figure $3 \mathrm{a}$, when DDM was mixed with different comonomers, AA, HEA and EGMAP, showed the highest polymerization rate and DC, followed by MAA, DDM, TEGDMA, MA, HEMA and MMA. AA, HEA, EGMAP and MAA contain either carboxylic acid $(-\mathrm{COOH})$, hydroxyl $(-\mathrm{OH})$, or phosphoric acid $\left(-\mathrm{OP}(\mathrm{OH})_{2}\right)$ groups, which belong to an electron-withdrawing group [15]. Furthermore, both AA and HEA are acrylate without a methyl $\left(-\mathrm{CH}_{3}\right)$ group on carbon-carbon double bond $(\mathrm{C}=\mathrm{C})$, but EGMAP and MAA are methacrylates, although both contain $-\mathrm{OP}(\mathrm{OH})_{2}$ and $-\mathrm{COOH}$ groups, respectively. Obviously, the acrylate group activates $\mathrm{C}=\mathrm{C}$, but the methyl group deactivates it. It is also evident that either methyl group on $\mathrm{C}=\mathrm{C}$ or alkyl group on the ester linkage can deactivate $\mathrm{C}=\mathrm{C}$ group, thus leading to slower polymerization (lower rate) and lower DC [15]. It was reported that the monomer containing phosphoric acid can increase the conversion and curing rate of the diacrylamide [10] and even the reaction rate can 
a
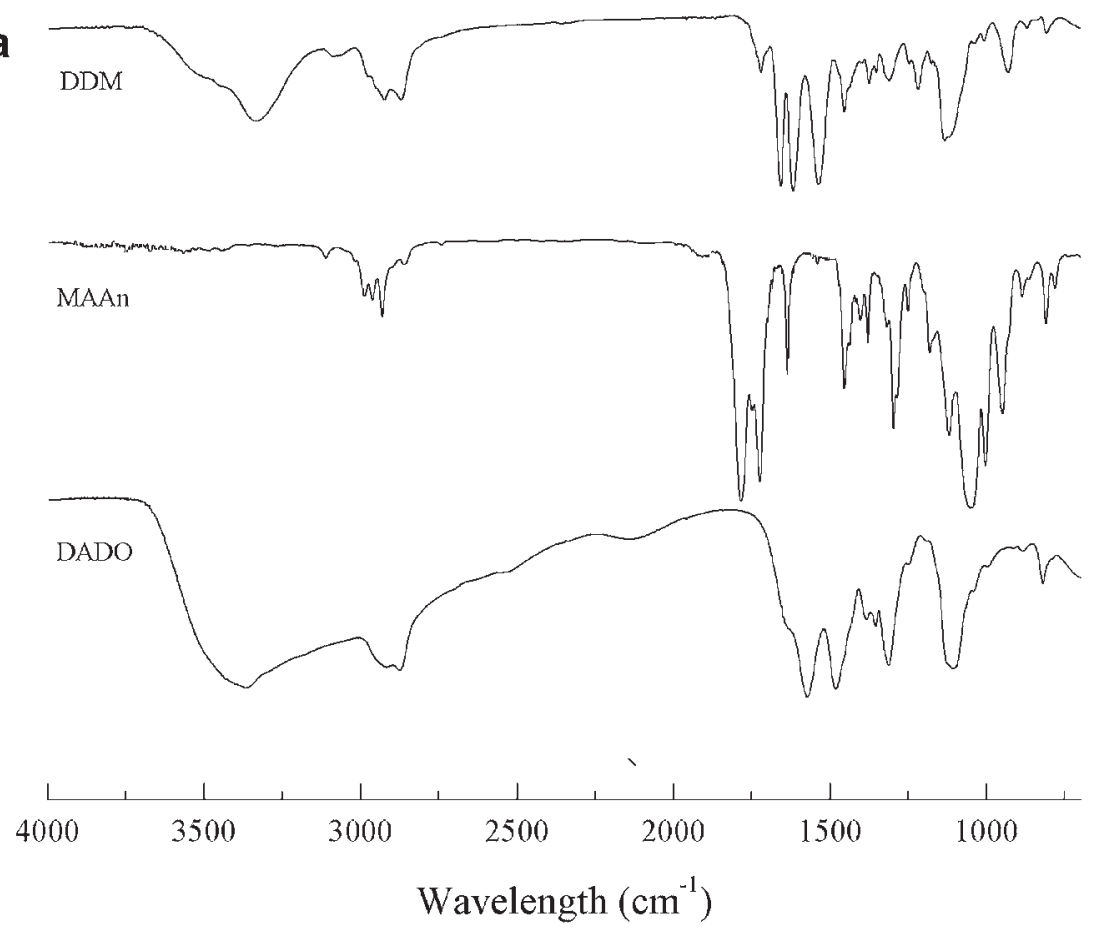

b $\mathrm{DDM}$
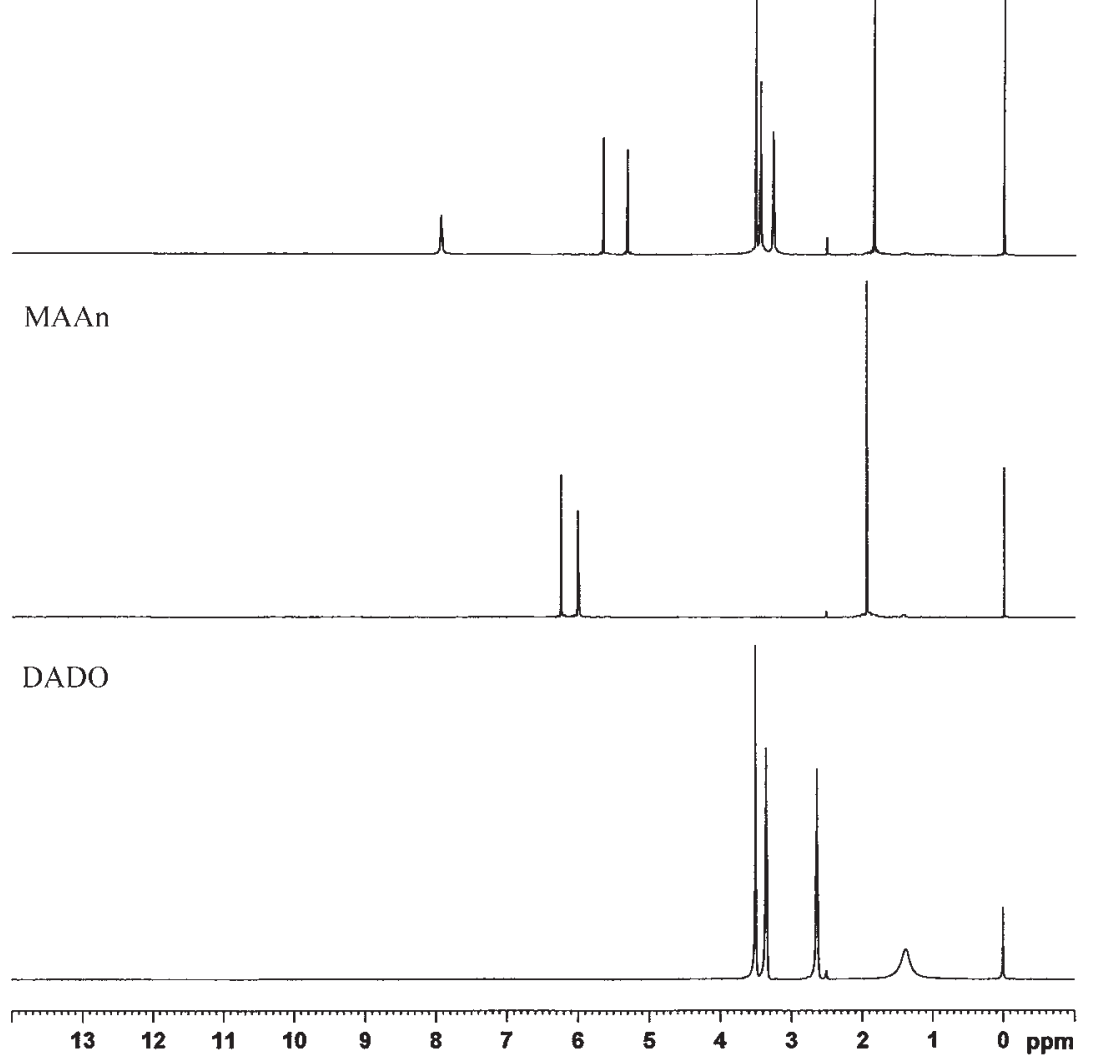

Figure 2. (a) FTIR and (b) ${ }^{1}$ HNMR spectra for DODA, MAAn and DDM. 
Table 1. Gel points of the tested resin formulations ${ }^{\mathrm{a}}$.

\begin{tabular}{lccc}
\hline & & \multicolumn{2}{c}{ Gel time (s) } \\
\cline { 3 - 4 } Name & Ratio & DDM & TEGDMA \\
\hline AA & $50 / 50$ & 40 & 138 \\
MAA & $50 / 50$ & 100 & 115 \\
HEA & $50 / 50$ & 75 & 86 \\
HEMA & $50 / 50$ & 318 & 202 \\
EGMAP & $50 / 50$ & 45 & 61 \\
MA & $50 / 50$ & 230 & 113 \\
MMA & $50 / 50$ & 420 & 238 \\
TEGDMA & $50 / 50$ & 190 & 71 \\
DDM & 100 & 170 & 190 \\
\hline
\end{tabular}

${ }^{\mathrm{a}}$ The observed gel points of the tested resins under visible light illumination at room temperature, where gel time $=$ the time when the resin starts to cure.

be enhanced in the presence of non-polymerizable organic phosphoric acid-containing reaction medium [11]. Hydrogen bonding (H-bonding) between amides on DDM and acid groups on AA, MAA and EGMAP may be another reason to show higher polymerization rate and DC. It was reported that the polymerization rate was higher for monomers that are capable of forming H-bonding [16-18]. These authors suggested that a higher dipole moment of the polymerizing medium reduced the termination rate $k_{\mathrm{t}}$, but $\mathrm{H}$-bonding raised the propagation rate $k_{\mathrm{p}}$, thus leading to an increased polymerization rate $[16,17]$. In our study, AA, MAA or EGMAP and even HEA can be regarded as either reaction medium or comonomer that are capable of forming strong H-bonding with DDM. Hence, AA showed the highest reaction rate, but DC and MMA showed the lowest. On the other hand, the case is quite different for TEGDMA. From figure 3b, when TEGDMA was mixed with the same comonomers, AA, TEGDMA, EGMAP and HEA, showed the highest polymerization rate, followed by MA, HEMA, MAA and MMA. HEA showed the highest DC, followed by AA $=$ MA > HEMA $>$ TEGDMA > EGMAP > MMA > MAA. There is no clear correlation from the viewpoint of molecular structure. It is known that DDM is an amide, whereas TEGDMA is an ester. When DDM is mixed with any monomer except for MA, MMA and TEGDMA, the H-bonding would form between DDM and these comonomers as amides in DDM provide H-bonds. In contrast, when TEGDMA was used, there was no H-bonding formed between TEGDMA and those comonomers because TEGDMA does not have any H-bonds on it. The DDM polymerization is more vulnerable to the surrounding groups whether they are electron-withdrawing or donating, and polar or non-polar.

Table 1 shows the observed gel time of the tested formulations. The gel time can be roughly used to evaluate the starting point of curing [8], although it is not as accurate as the conversion measured by FTIR. For the DDM-based resins, it was found that incorporation of the acid-containing and/or acrylate-containing monomer led to a shorter gel time or
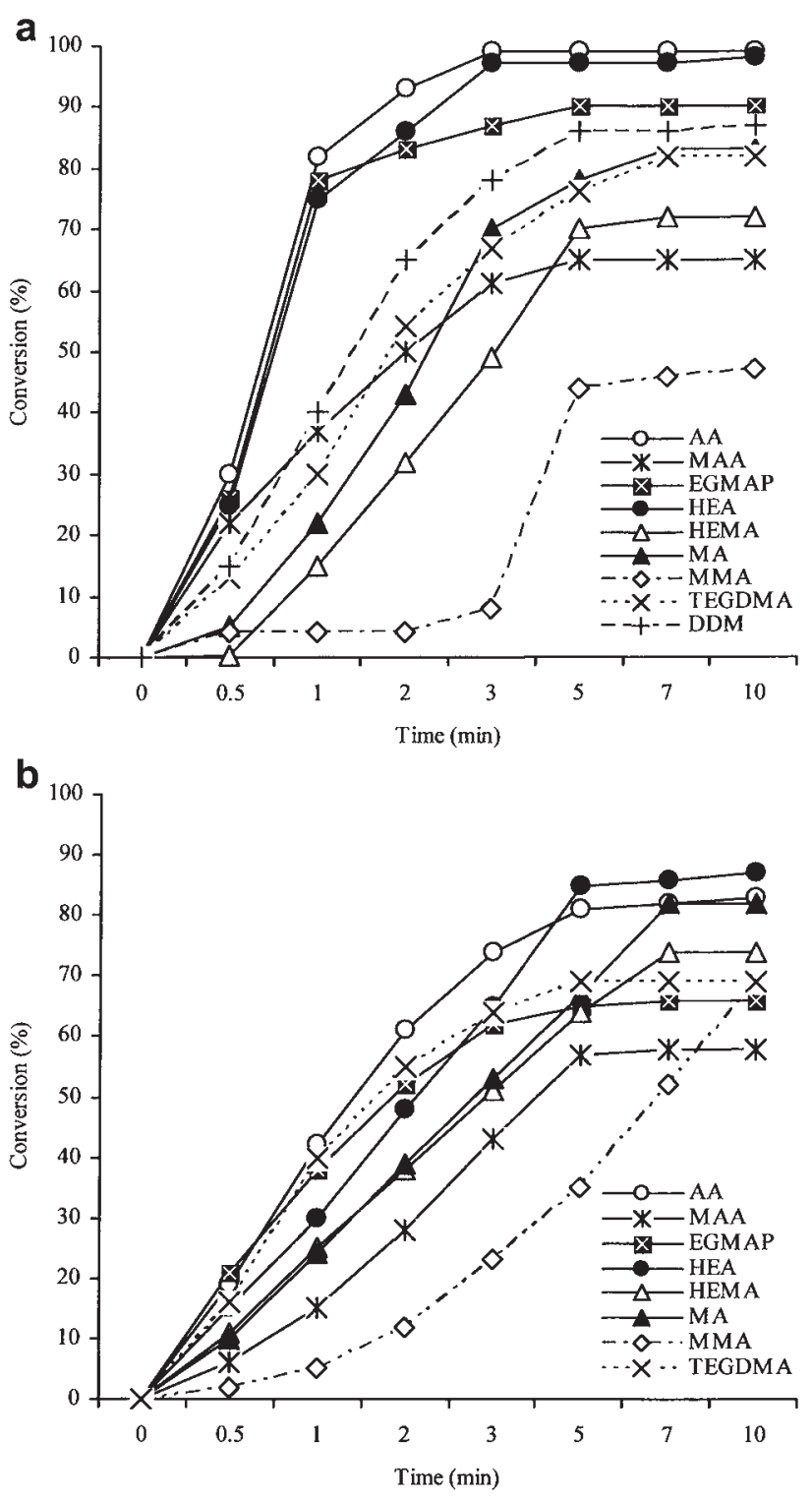

Figure 3. Conversion of (a) DDM- and (b) TEGDMA-based resins. The conversion values were calculated based on the absorbance in FTIR.

faster curing. On the other hand, the comonomers composed of methacrylate and/or methyl or alkyl groups next to the ester linkage showed the contradictory. The result from the gel time measurement was fairly consistent with that for the polymerization rate from the DC curves by FTIR, indicating that gel time may be used to roughly estimate the polymerization rate of the DDM-based resins in situ. In the case of the TEGDMAbased resins, however, the curing pattern did not follow the above discussion for the DDM-based system, although there exist some similarity. The electron-withdrawing group still shows some effect on polymerization rate, but the electrondonating group on $\mathrm{C}=\mathrm{C}$ or the ester linkage plays little role.

Figure 4 shows the CS of the DDM- and TEGDMA-based resins. Tables 2 and 3 show the detailed corresponding YS, 


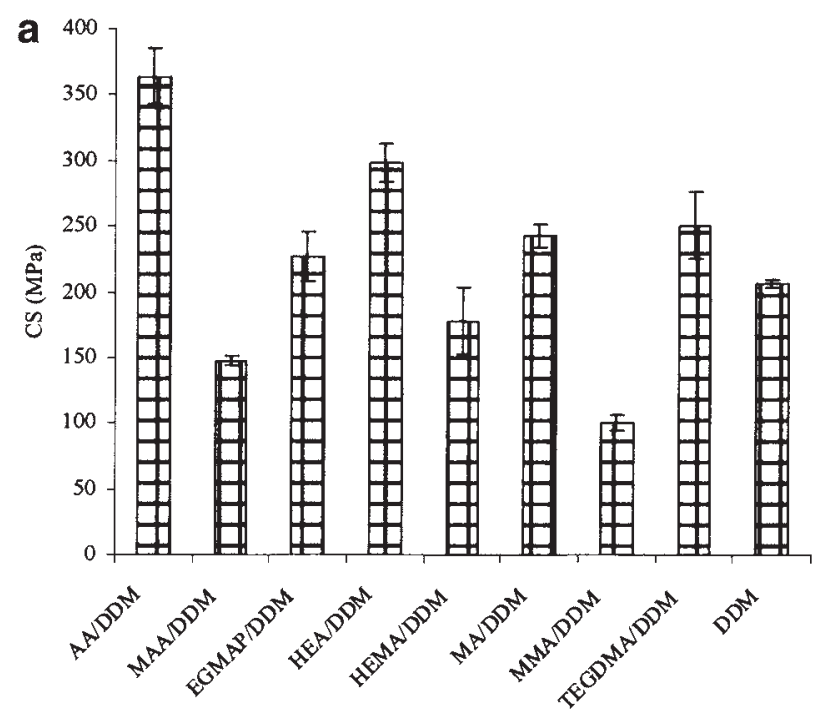

M, CS, T and ETY in the stress-strain curves. It is known that YS and $\mathrm{M}$ are often used to evaluate brittleness or stiffness of materials [19]. CS stands for break strength. T is defined as the area under stress-strain curve before break, which covers both elastic and plastic portions [19]. It represents consumed energy before break. ETY is defined as the area under stress-strain curve before plastic deformation [19], which only covers elastic portion and is closely related to YS and M. It represents consumed energy to yield. The data from figure 4 and table 2 strongly support the previously discussed viewpoints. For the DDM-based system (figure 4a), AA showed the highest CS, followed by HEA, MA, TEGDMA, EGMAP, DDM, HEMA, MAA and MMA. All the acrylate-containing comonomers showed much higher CS values than their corresponding methacrylate counterparts. The trend is similar to the gel time shown in table 1 . The lower CS values exhibited by EGMAP and MAA can be attributed to their lower DC. From table 2, AA, EGMAP and MAA showed the highest

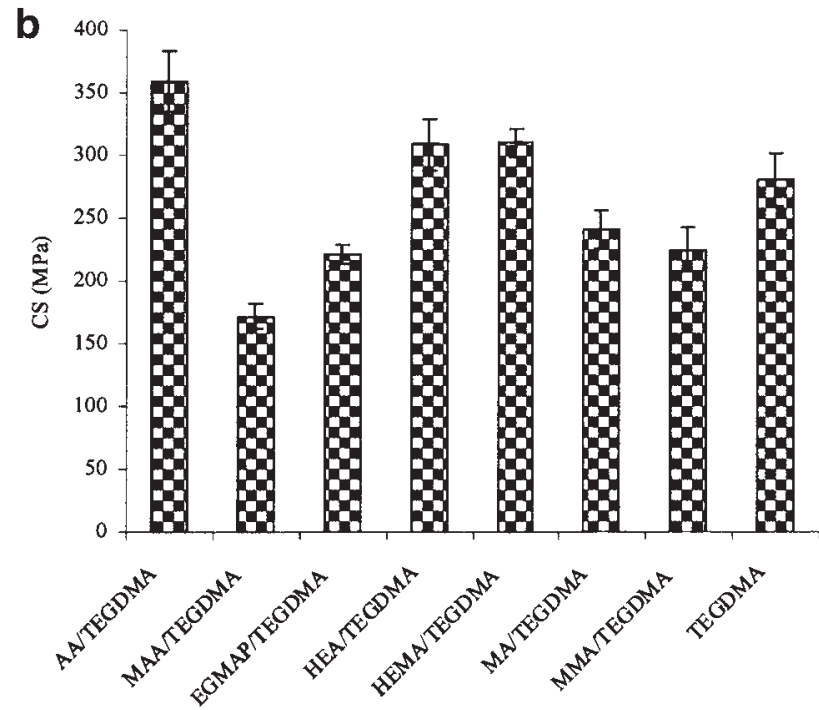

Figure 4. CS of (a) DDM- and (b) TEGDMA-based resins. YS, $M$ and ETY, indicating that these resins are much stiffer than the others, probably due to strong H-bonding between DDM and acid groups. HEA and MA were the softest resins with nearly no YS, very low M and low ETY. For T, AA was the highest, followed by EGMAP and TEGDMA, suggesting that these three resins are the toughest. The low YS and M of HEA, low CS of HEMA and MAA, and relatively low YS and $\mathrm{M}$ of DDM and MMA made these resins to show lower T. The lower T value exhibited by MAA can also be attributed to its lower DC due to the negative effect of methyl group on $\mathrm{C}=\mathrm{C}$.

For the TEGDMA-based resins (see figure $4 \mathrm{~b}$ and table 3 ), although AA was still the highest in all the measured strengths, EGMAP and MAA were not ranked as high in all the strengths as those shown in the DDM-based system. MAA was ranked the 2nd in YS, followed by HEMA, MMA, TEGDMA, EGMAP, MA and HEA. MAA was ranked the 1st in M, followed by AA, HEMA, MA, MMA, EGMAP, TEGDMA and TEA. HEMA was ranked the 2nd in CS,

Table 2. Compressive strengths of the DDM-based resins.

\begin{tabular}{|c|c|c|c|c|c|}
\hline Material & YS (MPa) & $\mathrm{M}(\mathrm{GPa})$ & $\mathrm{CS}(\mathrm{MPa})$ & $\mathrm{T}(\mathrm{KNmm})$ & ETY $(\mathrm{Nmm})$ \\
\hline AA & $159.7(7.9)$ & $4.09(0.19)$ & $364.2(21)$ & $8.57(0.33)$ & $425.3(16)$ \\
\hline MAA & $108.8(8.5)^{\mathrm{a}, 1}$ & $2.93(0.13)$ & $147.0(3.6)$ & $2.50(0.09)^{\mathrm{d}}$ & $297.3(14)$ \\
\hline EGMAP & $112.6(3.8)^{\mathrm{a}}$ & $2.38(0.05)$ & 226.7 (12) & $4.22(0.17)^{\mathrm{e}}$ & $348.8(7.1)$ \\
\hline HEA & $7.8(0.9)$ & $0.85(0.08)$ & $298.1(15)$ & $2.83(0.09)$ & $36.4(5.0)$ \\
\hline HEMA & $43.7(3.7)^{b}$ & $1.27(0.10)^{\mathrm{c}}$ & $177.7(21)$ & $3.14(0.31)$ & $94.7(6.0)^{f}$ \\
\hline MA & $4.0(0.33)$ & $0.30(0.01)$ & $242.5(8.5)$ & $1.71(0.24)$ & $24.3(4.5)$ \\
\hline MMA & $40.9(3.2)^{b}$ & $1.02(0.11)$ & $100.5(5.5)$ & $2.40(0.05)^{\mathrm{d}}$ & $119.8(10)$ \\
\hline TEGDMA & $63.3(2.5)$ & $1.63(0.03)$ & $264.5(7.7)$ & $4.18(0.06)^{\mathrm{e}}$ & $144.4(5.2)$ \\
\hline DDM & $31.9(2.7)$ & $1.18(0.06)^{\mathrm{c}}$ & 206.9 (3.1) & $2.20(0.17)$ & $102.9(7.9)^{\mathrm{f}}$ \\
\hline
\end{tabular}

${ }^{1}$ The formulations are the same as those described in table $1 . \mathrm{YS}=$ yield compressive strength, $\mathrm{CM}=$ compressive modulus, $\mathrm{CS}=$ ultimate compressive strength, $\mathrm{T}=$ toughness which is the area under $\sigma-\varepsilon$ curve in compression, ETY $=$ energy to yield.

Entries are the mean values with standard deviations in parentheses and the mean values with the same letter in each category were not significantly different $(P>0.05)$. Specimens were tested after conditioning at $37^{\circ} \mathrm{C}$ for $24 \mathrm{~h}$. 
Table 3. Compressive strengths of the TEGDMA-based resins.

\begin{tabular}{|c|c|c|c|c|c|}
\hline Material & YS (MPa) & $\mathrm{M}(\mathrm{GPa})$ & $\mathrm{CS}(\mathrm{MPa})$ & $\mathrm{T}(\mathrm{KNmm})$ & ETY (Nmm) \\
\hline AA & $133.3(6.5)$ & $2.75(0.29)$ & $358.9(24)$ & $7.68(0.36)$ & $425.3(22)$ \\
\hline MAA & $121.1(7.1)$ & $3.26(0.13)$ & $171.4(9.9)$ & $3.16(0.55)^{\mathrm{h}}$ & $342.1(33)$ \\
\hline EGMAP & $73.0(4.3)^{\mathrm{a}}$ & $1.71(0.08)^{\mathrm{c}}$ & $221.5(7.3)^{\mathrm{e}}$ & $3.91(0.09)$ & $195.1(16)$ \\
\hline HEA & $43.4(8.7)$ & $1.34(0.19)$ & $308.5(20)^{\mathrm{f}}$ & $4.08(0.43)^{i, j}$ & $81.5(13)$ \\
\hline HEMA & $90.2(13)^{b}$ & $2.23(0.23)^{\mathrm{d}}$ & $310.1(11)^{\mathrm{f}}$ & $5.47(0.26)$ & $253.1(21)^{\mathrm{k}}$ \\
\hline MA & $65.1(2.9)^{\mathrm{a}}$ & $2.07(0.07)^{\mathrm{d}}$ & $240.8(15)^{\mathrm{g}}$ & $4.04(0.42)^{\mathrm{i}}$ & $134.8(6.8)$ \\
\hline MMA & $86.9(3.9)^{b}$ & $1.82(0.14)^{\mathrm{c}}$ & $225.1(18)^{\mathrm{e}, \mathrm{g}}$ & $3.31(0.36)^{\mathrm{h}}$ & $272.5(25)^{\mathrm{k}, 1}$ \\
\hline TEGDMA & $75.4(9.5)$ & $1.56(0.21)$ & $280.1(21)$ & $4.19(0.45)^{\mathrm{j}}$ & $281.8(19)^{1}$ \\
\hline
\end{tabular}

${ }^{1}$ The formulations are the same as those described in table $1 . \mathrm{YS}=$ yield compressive strength, $\mathrm{CM}=$ compressive modulus, CS = ultimate compressive strength, $\mathrm{T}=$ toughness, which is the area under $\sigma-\varepsilon$ curve in compression, ETY = energy to yield.

Entries are mean values with standard deviations in parentheses and the mean values with the same letter in each category were not significantly different $(P>0.05)$. Specimens were tested after conditioning at $37^{\circ} \mathrm{C}$ for $24 \mathrm{~h}$.

followed by HEA, TEGDMA, MA, MMA, EGMAP and MAA. HEMA was ranked the 2nd in $T$, followed by TEGDMA, HEA, MA, EGMAP, MMA and MAA. MAA was ranked as the 2nd in ETY, followed by TEGDMA, MMA, HEMA, EGMAP, MA and HEA. Except for AA and MAA, there is no negative influence from methyl group on $\mathrm{C}=\mathrm{C}$, if one compares HEA with HEMA or MA with MMA, unlike in the DDM-based system. The effect of the electronwithdrawing group was not significant either if one compares AA, MAA and EGMAP with HEA, HEMA, MA and MMA. The effect of the electron-donating group was not significant at all, which can be easily observed between HEA and HEMA or between MA and MMA (no difference in CS at all). Obviously there is no clear pattern to follow the strength ranking in the TEGDMA-based resins, suggesting that there is no special interaction between TEGDMA and acid-containing comonomer including AA, MAA and EGMAP.

\section{Conclusions}

In situ visible light polymerization of the dimethacrylamidebased resins was studied. Generally speaking, when dimethacrylamide was copolymerized with the monomer having an electron-withdrawing group and/or an acrylate group, shorter gel time, faster polymerization rate, higher DC and higher mechanical strength were obtained and vise versa. In contrast, the TEGDMA-based system did not show a clear pattern. This may be attributed to the molecular structure difference between dimethacrylamide and dimethacrylate, because the former is a H-bond provider, but the latter is not. The H-bonds between (meth)acrylamide and organic acid groups may be responsible for the exhibited higher strengths. It is suggested that monomers containing electronwithdrawing and acrylate groups would favour in situ curing of (meth)acrylamide or di(meth)acrylamide. Future study will include the investigation, which specific wavelength of the visible light may play a major role in the acceleration of in situ light polymerization of di(meth)acrylamide-based resin system, if possible, and to study how to maximize the polymerization rate and conversion under this specific wavelength.

\section{Acknowledgements}

This study was partially sponsored by IUPUI BBRC.

\section{References}

[1] Allen N S 2010 Photochemistry and photophysics of polymeric materials, 1st edn (NJ: John Wiley \& Sons, Inc.)

[2] Xia P, Zhang J, Damur F and Lalevee P 2015 J. Prog. Polym. Sci. 4132

[3] Ratner B D, Hoffman A S, Schoen F J and Lemons J E 2013 Biomaterials science: an introduction to materials in medicine, 3rd edn (Oxford, UK: AP, Elsevier Inc.)

[4] Moszner N and Salz U 2001 Prog. Polym. Sci. 26535

[5] Lin B A, Jaffer F, Duff M D, Tang Y W and Santerre J P 2005 Biomaterials 264259

[6] Cai K, Delaviz Y, Banh M, Guo Y and Santerre J P 2014 Dent. Mater. 30848

[7] Bettencourt A F, Neves C B, de Almeida M S, Pinheiro L M, Arantes e Oliveira S, Lopes L P et al 2010 Dent. Mater. 26 e171

[8] Moszner N, Zeuner F, Angerman J, Fischer U K and Rheinberger V 2003 Macromol. Mater. Eng. 288621

[9] Ullrich G, Burscher P, Saltz U, Moszner N and Liska R 2006 J. Polym. Sci. Part A: Polym. Chem. 44115

[10] Catel Y, Degrange M, Pluart L L, Madec P J, Pham T N, Chen F et al 2009 J. Polym. Sci. Part A: Polym. Chem. 47 5258

[11] Besse V, Pluart L, Cook W D, Pham T-N and Madec P-J 2013 J. Polym. Sci. Part A: Polym. Chem. 51149

[12] Xie D, Park J-G, Zhao J and Turner C 2007 J. Biomater. Appl. 2233

[13] Wang G, Culbertson B M, Xie D and Seghi R 1999 J. M. S. Pure Appl. Chem. A36 225 
[14] Weng Y, Howard L, Chong V J, Guo X, Gregory R L and Xie D 2012 J. Mater. Sci. Mater. Med. 23 1553

[15] Klein D R 2012 Organic chemistry, 2nd edn (NJ, USA: John Wiley \& Sons, Inc.)

[16] Jansen J F G A, Dias A, Dorschu D M and Coussens B 2002 Macromolecules 357529
[17] Jansen J F G A, Dias A, Dorschu D M and Coussens B 2003 Macromolecules 363861

[18] Lee T Y, Roper T M, Johnson E S, Guymon C A and Hoyle C E 2004 Macromolecules 373659

[19] Callister Jr W D and Rethwisch D G 2013 Materials science and engineering: an introduction, 9th edn (NJ, USA: John Wiley \& Sons, Inc.) 